\title{
Lattice-mismatched GaAsP Solar Cells Grown on Silicon by OMVPE
}

\author{
John F. Geisz, J.M. Olson, M.J. \\ Romero, C.S. Jiang, A.G. Norman \\ National Renewable Energy Laboratory
}

at the

4th World Conference on Photovoltaic Energy Conversion, 2006, Hawaii

Presented at the 2006 IEEE 4th World Conference on Photovoltaic Energy Conversion (WCPEC-4) held May 7-12, 2006 in Waikoloa, Hawaii. 


\section{Disclaimer and Government License}

This work has been authored by Midwest Research Institute (MRI) under Contract No. DE-AC36-99G010337 with the U.S. Department of Energy (the "DOE"). The United States Government (the "Government") retains and the publisher, by accepting the work for publication, acknowledges that the Government retains a non-exclusive, paid-up, irrevocable, worldwide license to publish or reproduce the published form of this work, or allow others to do so, for Government purposes.

Neither MRI, the DOE, the Government, nor any other agency thereof, nor any of their employees, makes any warranty, express or implied, or assumes any liability or responsibility for the accuracy, completeness, or usefulness of any information, apparatus, product, or process disclosed, or represents that its use would not infringe any privately owned rights. Reference herein to any specific commercial product, process, or service by trade name, trademark, manufacturer, or otherwise does not constitute or imply its endorsement, recommendation, or favoring by the Government or any agency thereof. The views and opinions of the authors and/or presenters expressed herein do not necessarily state or reflect those of MRI, the DOE, the Government, or any agency thereof. 


\section{Motivation}

- Monolithic, two (or more) junction single crystal solar cell on $\mathrm{Si}$

- Old idea (SERI 1980's)

Advantages of silicon substrates:

- Excellent solar cell material

- Lower cost than III-V or Ge

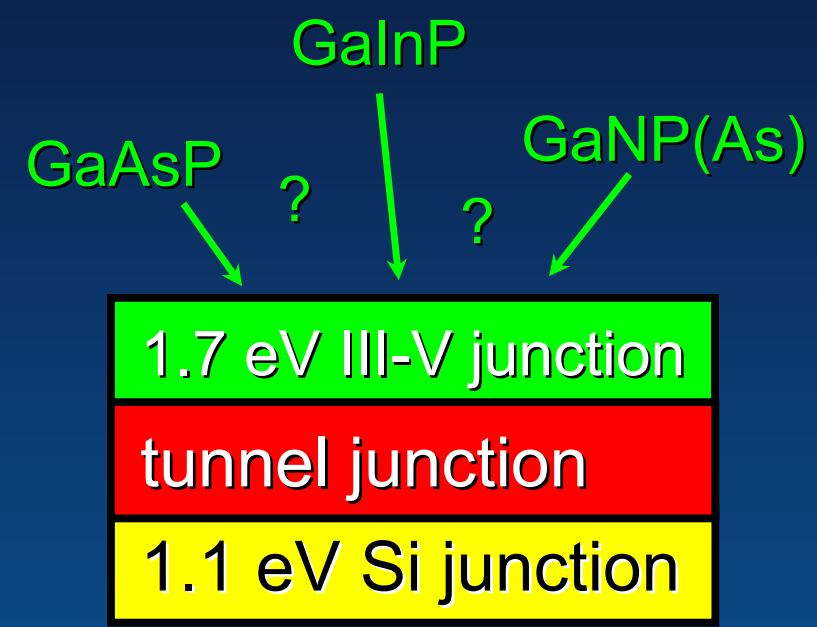

- Mature Si technology

- Mechanically robust

- Two-junction cell using Si bottom junction is nearly optimal theoretical efficiency

- $34 \%$ efficiency at 1 sun AMO

- $44 \%$ efficiency at 500 sun AM1.5G 


\section{Challenges for III-V on Si growth}

- Silicon oxides

- Surface contamination

- Antiphase domains

- Etching by sources

- Interdiffusion

- Lattice-mismatch

- Thermal expansion mismatch

New developments

- Improved characterization techniques

- Better understand for III-V nucleation

- Lattice-matched GaNPAs materials 


\section{P-on-N GaAsP Cell Structure}

- III-V grown by OMVPE

- Smooth GaP nucleation on $\mathrm{Si}$

- Step graded GaAsP buffer to reduce dislocations

- Constant lattice GaAsP junction with GalnP passivation

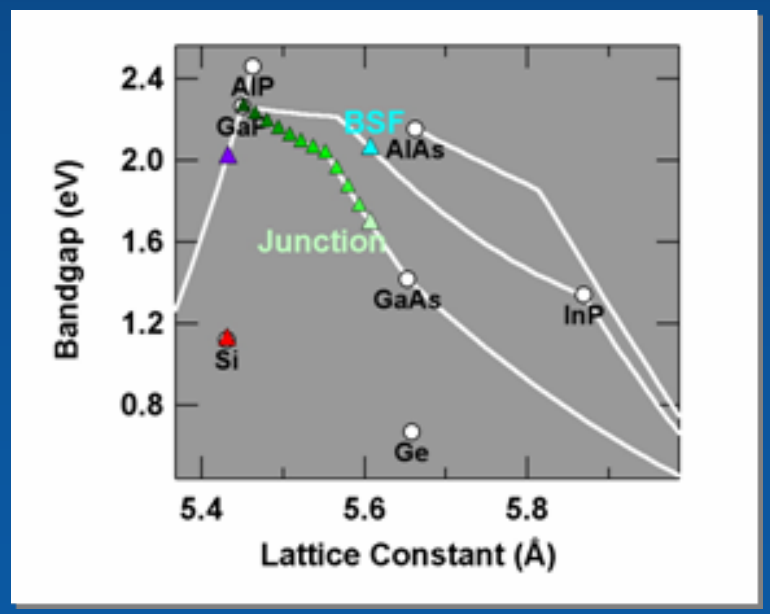

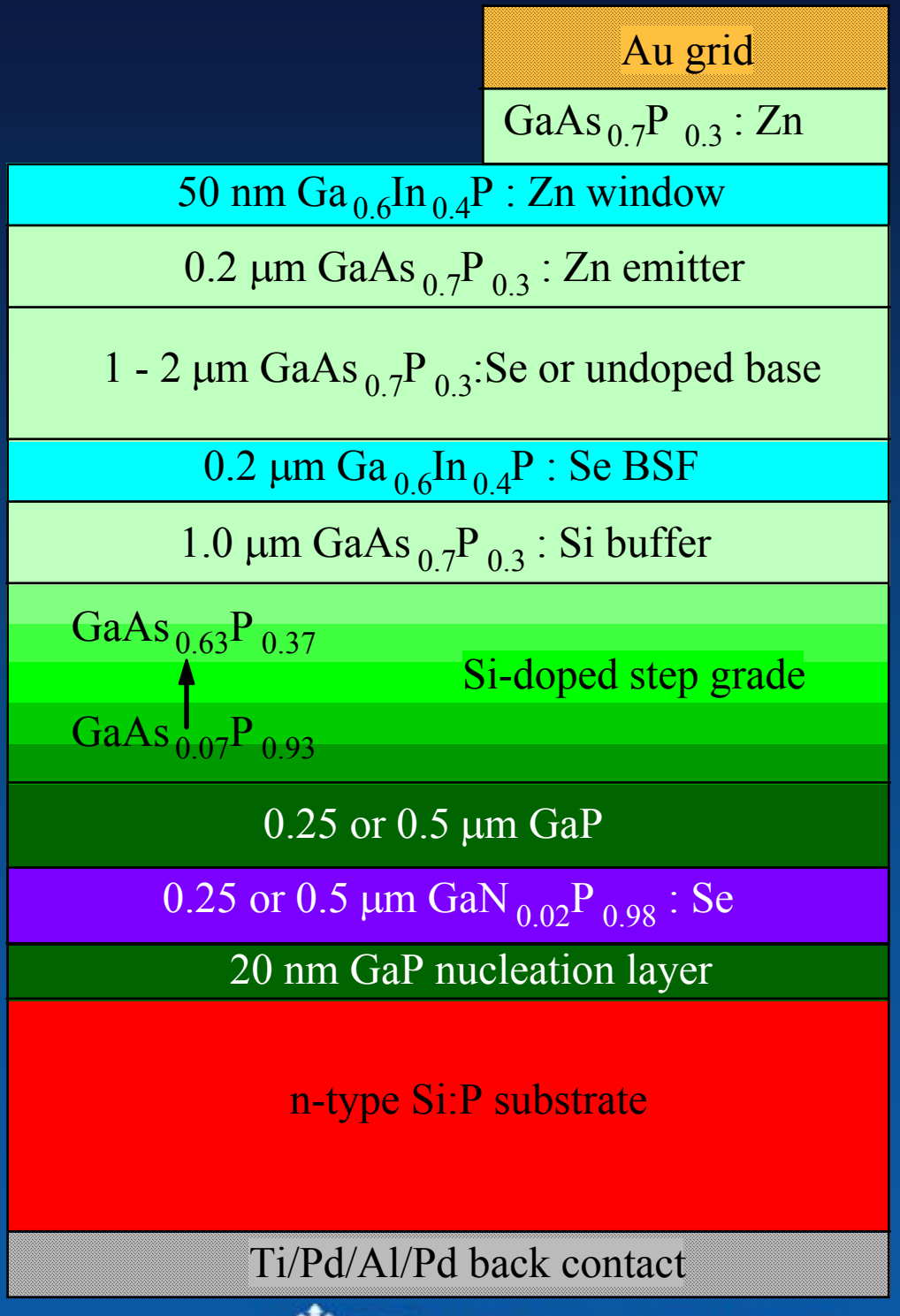




\section{GaP Nucleation on Si}

- Antiphase domains during GaP nucleation revealed by AFM - Growth of LM GaNP smoothes surface and reduces APD

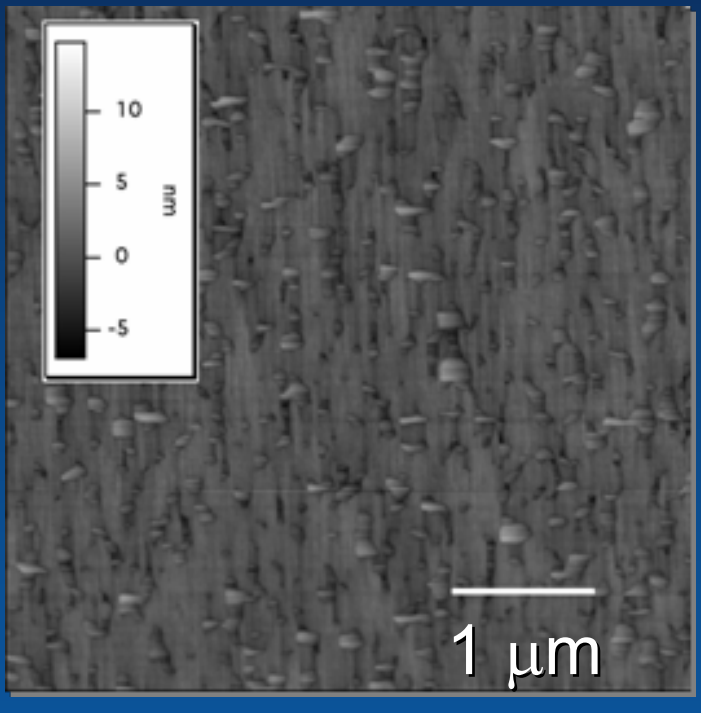

$5.7 \mathrm{~nm} \mathrm{GaP}$ $\mathrm{RMS}=1.2 \mathrm{~nm}$

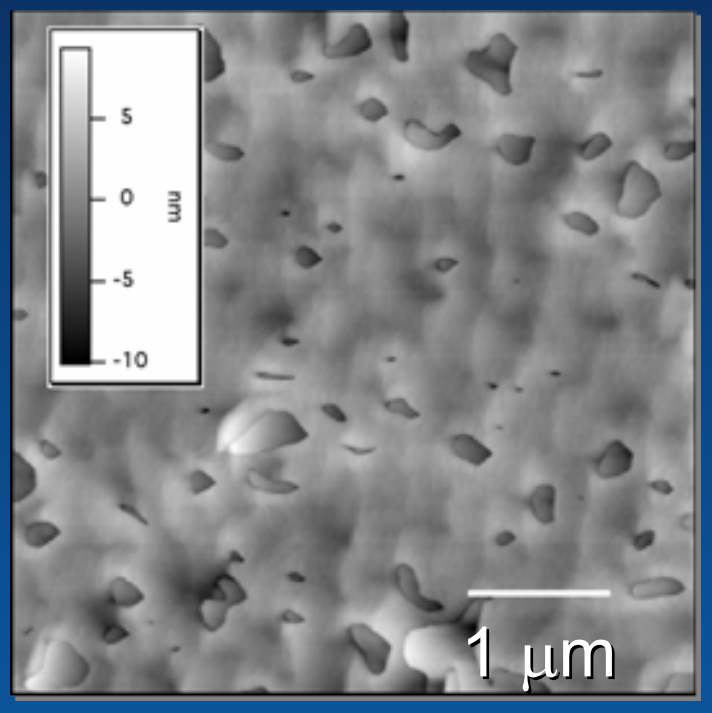

$17 \mathrm{~nm} \mathrm{GaP}$ $33 \mathrm{~nm}$ GaNP RMS $=1.9 \mathrm{~nm}$

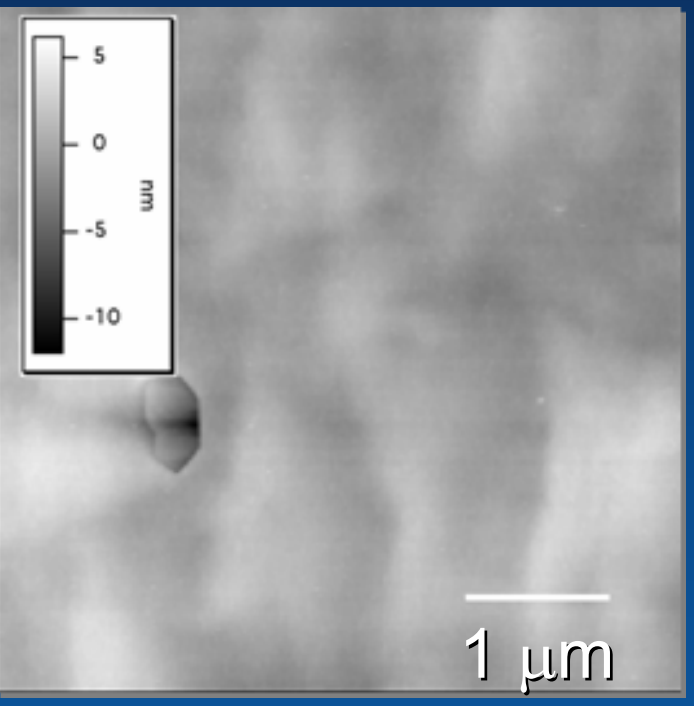

$18 \mathrm{~nm} \mathrm{GaP}$ $403 \mathrm{~nm}$ GaNP $\mathrm{RMS}=0.4 \mathrm{~nm}$ 


\section{Step graded buffer layer}

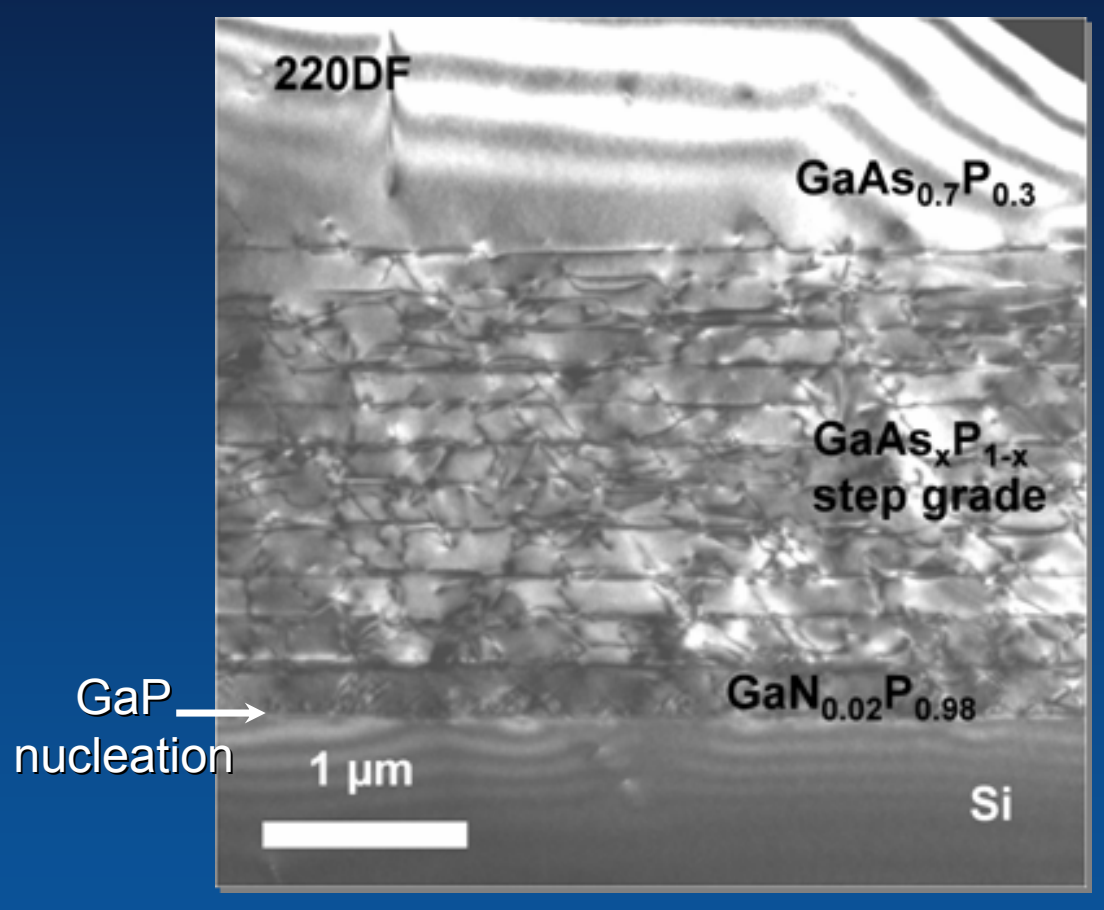

- 0.25 or $0.5 \mu \mathrm{m}$ $\mathrm{GaAs}_{x} \mathrm{P}_{1-\mathrm{x}}$ steps

- Composition change $\Delta x=0.07$ per step

- 3.75 or $6.5 \mu \mathrm{m}$ total buffer thickness

- Many dislocations in grade, but few in active layers

Cross-sectional TEM 


\section{Composition and Strain}

- Grown under compression because growing epilayers have larger lattice constant

- Relaxes at $\mathrm{T}_{\mathrm{g}}$ above critical thickness

- Cools toward tension

- Residual compressive strain at $T_{g}$ results in less tension at RT (no cracks)

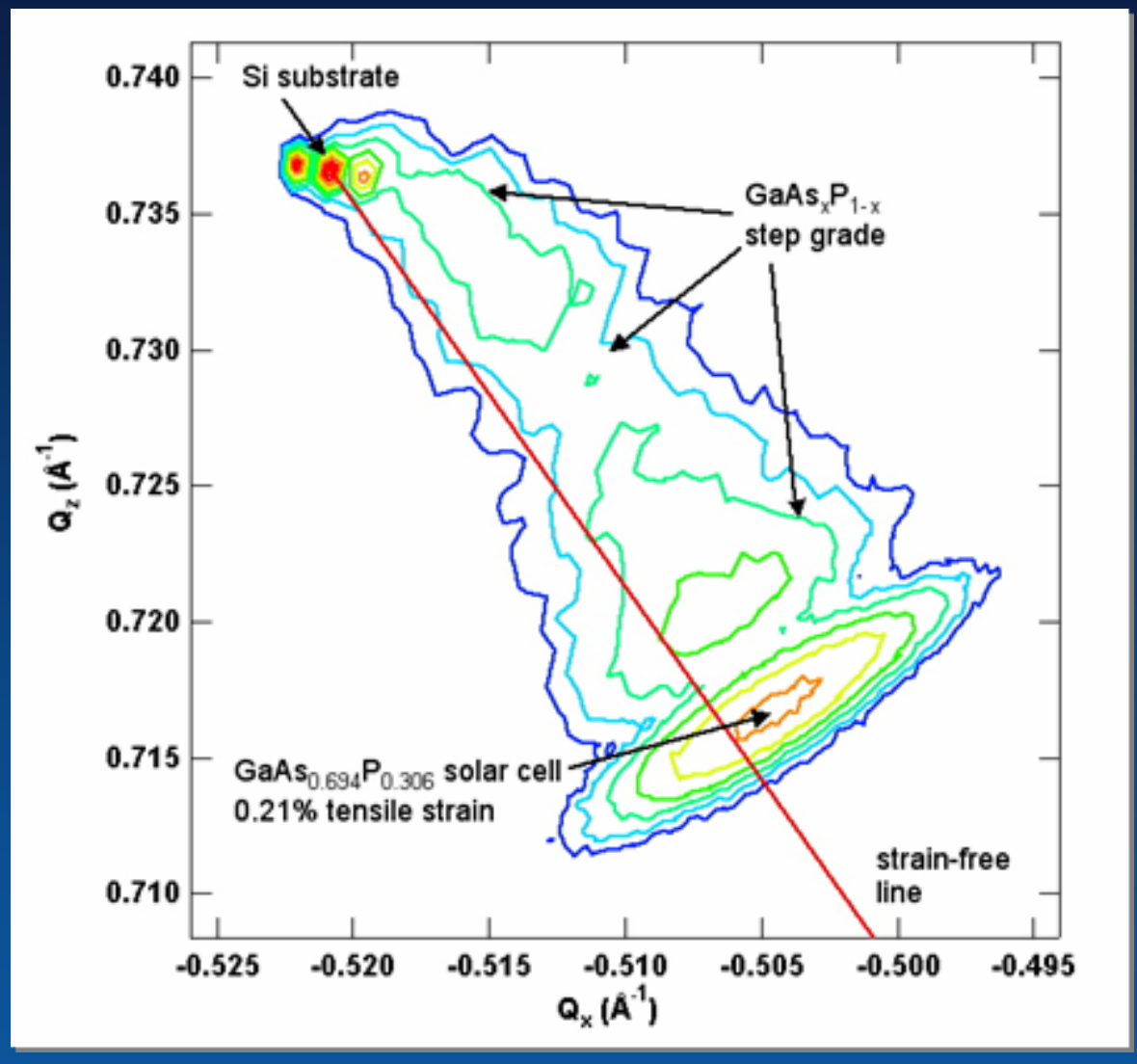

\section{X-ray diffraction 224GI reflection RSM}




\section{Threading Dislocations}

- Electron-Beam-Induced Current (EBIC)

- $9 \times 10^{7}-1 \times 10^{8} \mathrm{~cm}^{-2}$ threading dislocations

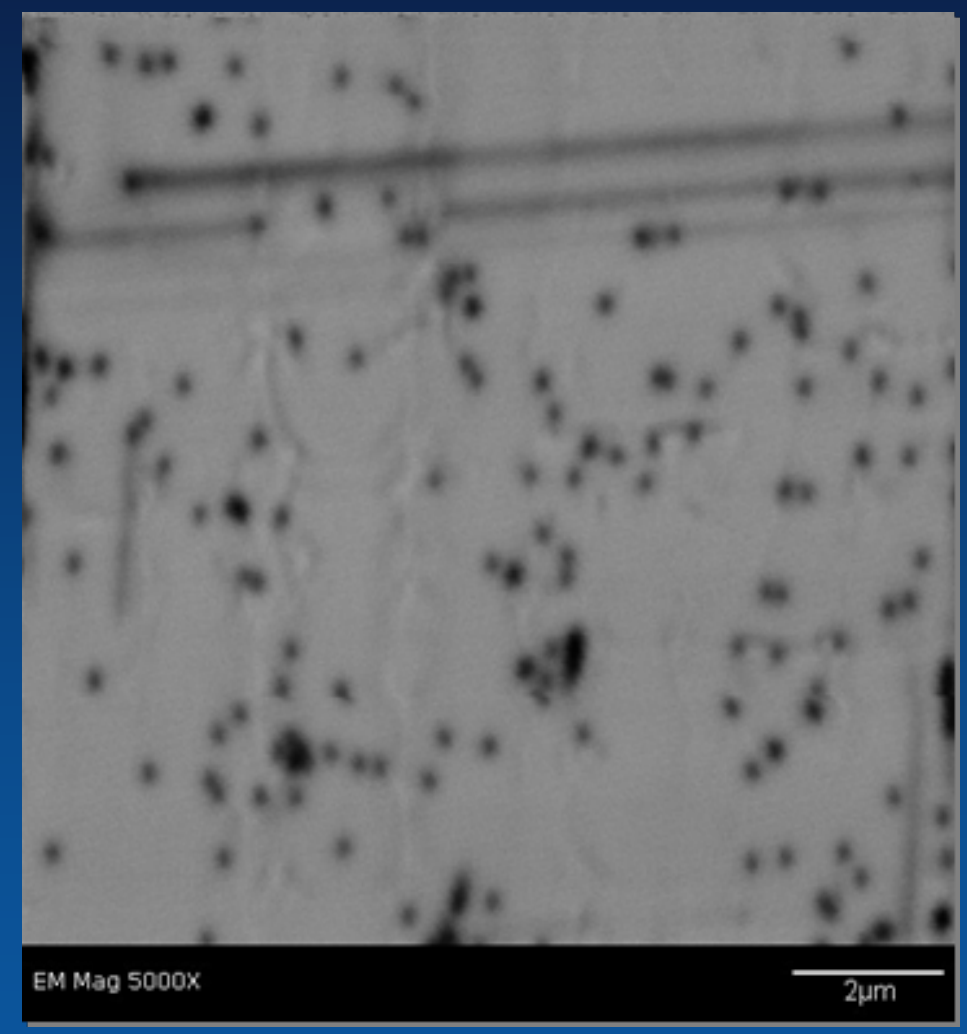

Plan-view EBIC 


\section{GaAsP/Si Device Performance}
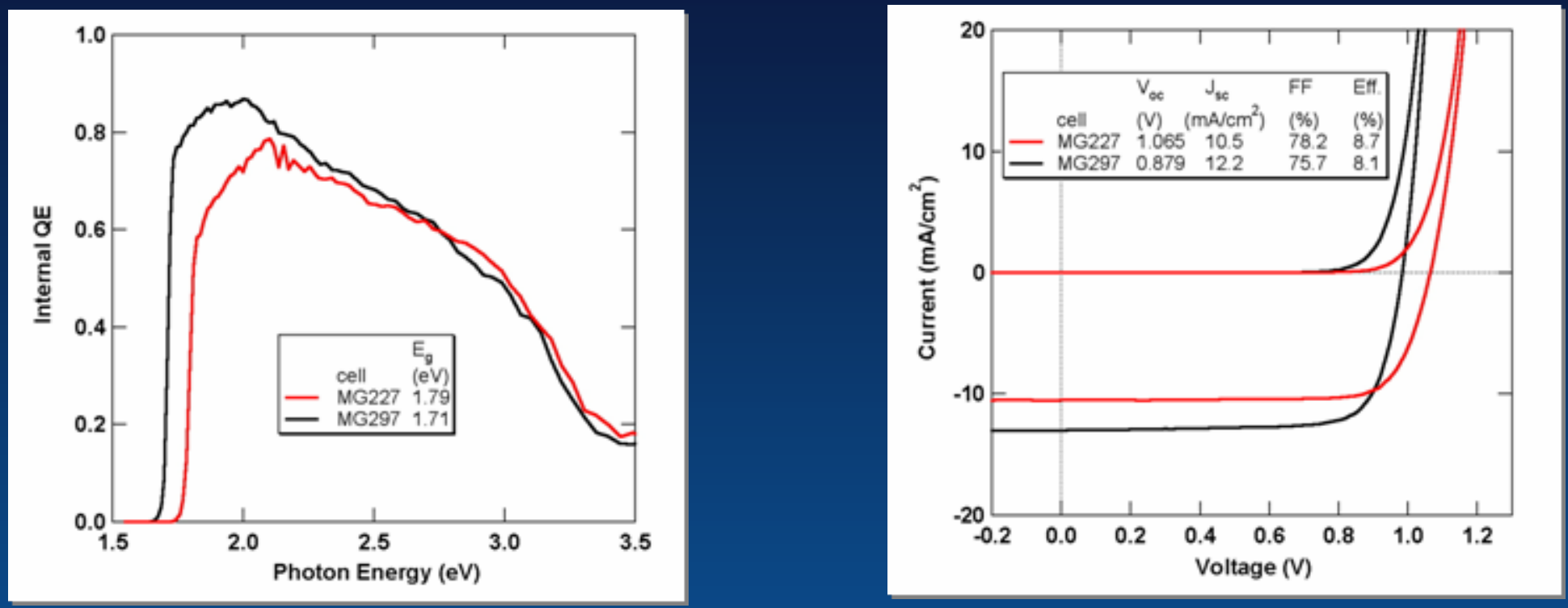

- Single-junction 8.7\% efficiency AM1.5G w/o AR coat

- Increase $J_{s c}$ with lower $E_{g}$ (need about $20 \mathrm{~mA} / \mathrm{cm}^{2}$ for 2-junction current matching)

- Improve QE with better passivation, thinner window 


\section{Literature Comparison}

GaAsP/GaAs $10^{6} \mathrm{~cm}^{-2}$ Vernon, 19th PVSC, (1987),108 Wanlass, 19th PVSC, (1987), 530

\section{$V_{o c}$ is excellent} measure of quality for mismaitched solar cells

GaAs/SiGe/Si $10^{6} \mathrm{~cm}^{-2}$ Ringel, Prog. PV 10, (2002), 417

AlGaAs/Si $\sim 10^{7} \mathrm{~cm}^{-2}$

Soga, J.Appl. Phys., 78, (1995), 4196
LM GaNP(As)/Si or GaP Geisz, 31st PVSC, (2005), 695

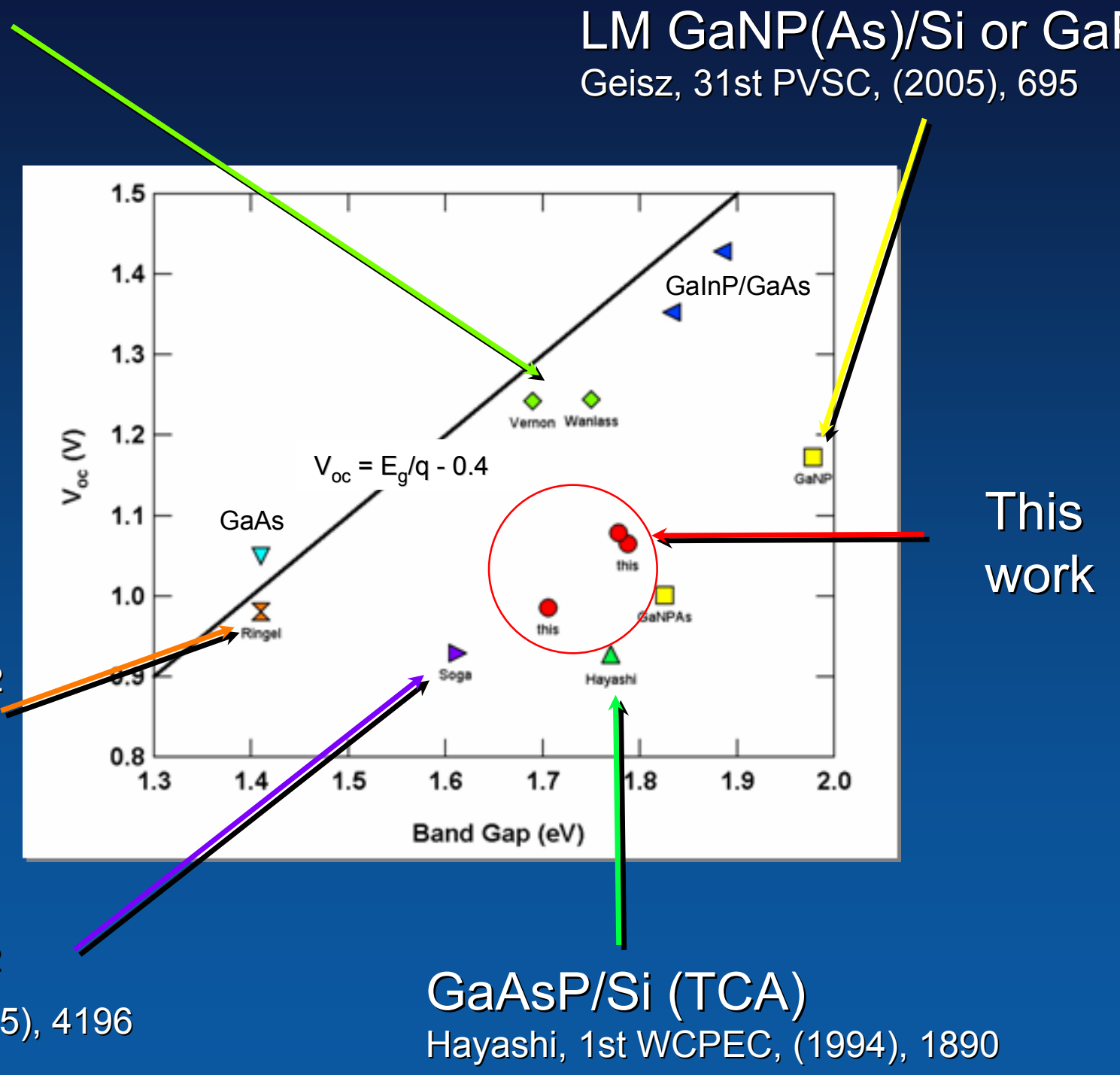




\section{Conclusions}

- Lattice-mismatched GaAsP solar cells grown on $\mathrm{Si}$

- Compositional step grade reduced dislocations to $\sim 10^{8} \mathrm{~cm}^{-2}$

- $V_{\text {oc }}$ not ideal, but comparable to best III-V grown on Si with transparent buffer

- Diffusion lengths better than LM GaNP

- Want to reduce dislocations to $10^{6} \mathrm{~cm}^{-2}$ 


\section{Extra Slides}




\section{Thermal Expansion}

- Can measure strain at RT with XRD

- Would like to measure strain during growth

- Can calculate strain state at Tg assuming

- change in in-plane lattice constant of epilayers constrained by thick $\mathrm{Si}$

- no relaxation upon cool-down

- Linear coefficients of thermal expansion $\left(\mathrm{K}^{-1}\right)$

- Si: $\quad 3.7 \times 10^{-6}$

- GaP: $\quad 5.3 \times 10^{-6}$

- GaAs: $\quad 6.8 \times 10^{-6}$

- GaN: $6 \times 10^{-6}$ ?

- InAs: $\quad 5.2 \times 10^{-6}$

- Scales with Tg

- Biaxial strain energy $U=Y \varepsilon_{x}{ }^{2} t$

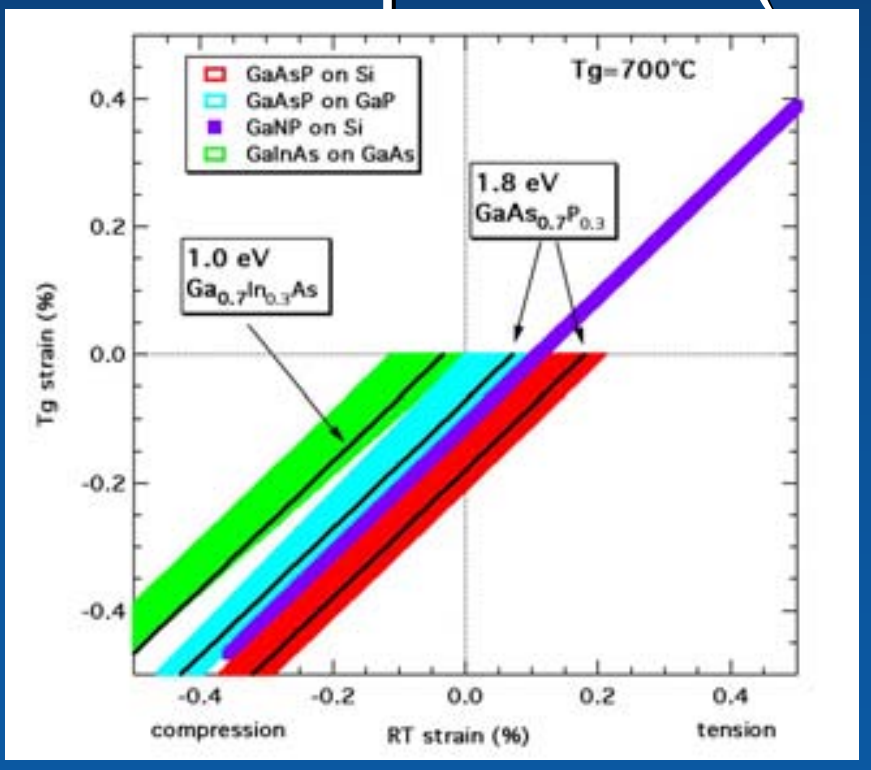




\section{Cracking from Tensile Strain on Si}
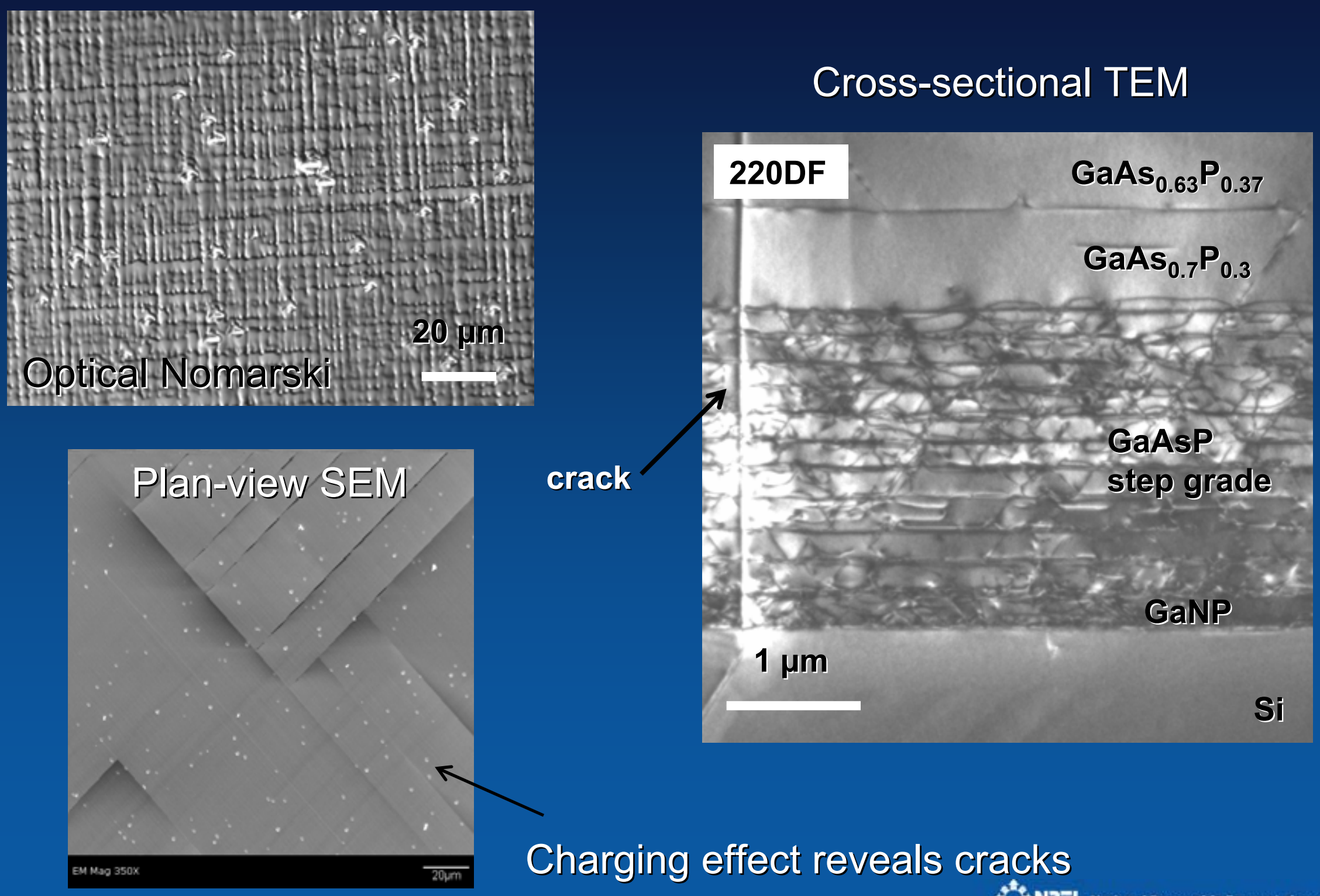

F NREL National Renewable Energy Laboratory 


\section{Si junction}

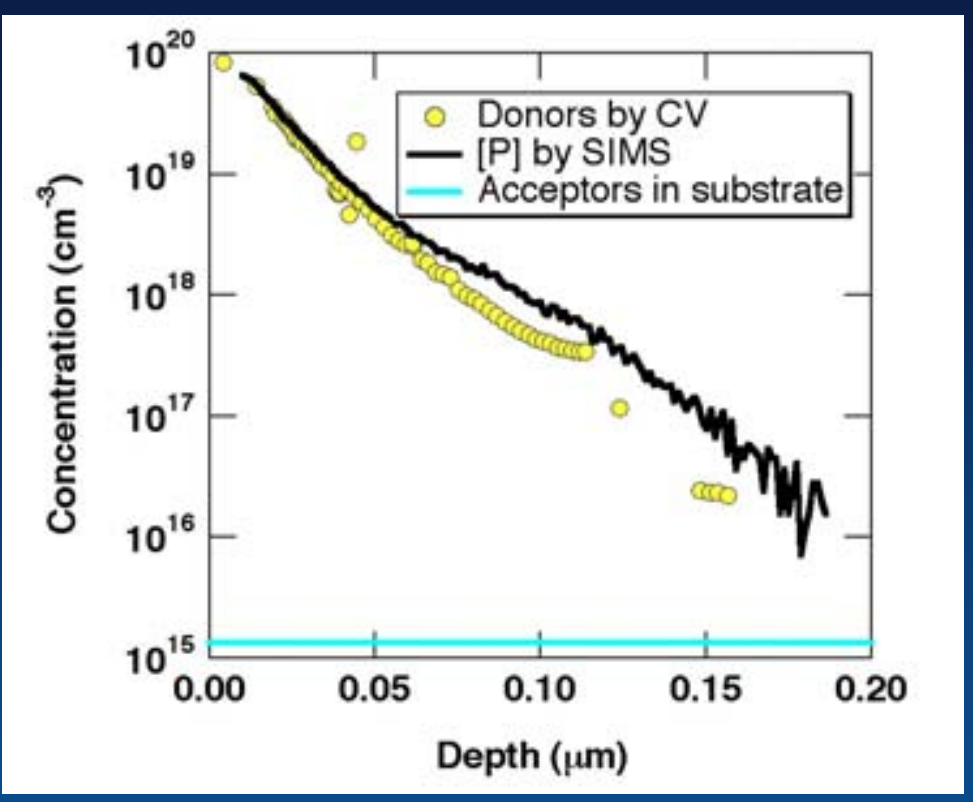

- Under current growth conditions, more $\mathrm{P}$ than Ga diffusion into $\mathrm{Si}$ from GaP

- Creates n-type emitter in silicon

- Emitter passivation from GaP if no interface defects

- $\mathrm{V}_{\text {oc }}$ of silicon junction $\sim 535 \mathrm{mV}$

- Currently using CZ Si, but float-zone may be better

- BSF from annealed Al contact

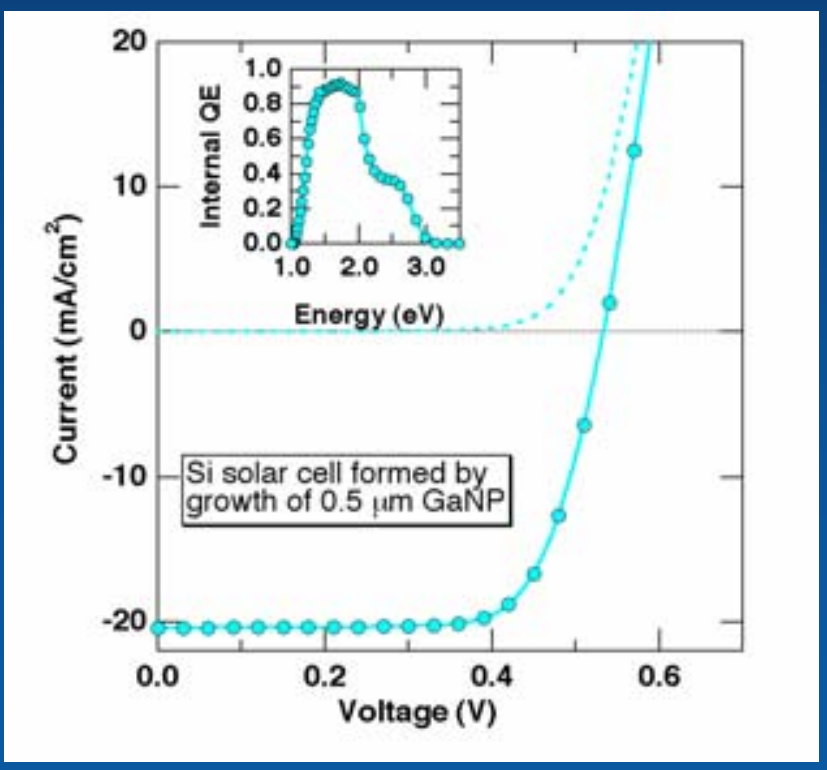




\section{GaNPAs/Si Tandem Solar Cell Results}

- Working tandem

- Good current in Si junction, but Voc could be a little better

- GaNPAs delivers half the current necessary to current match tandem
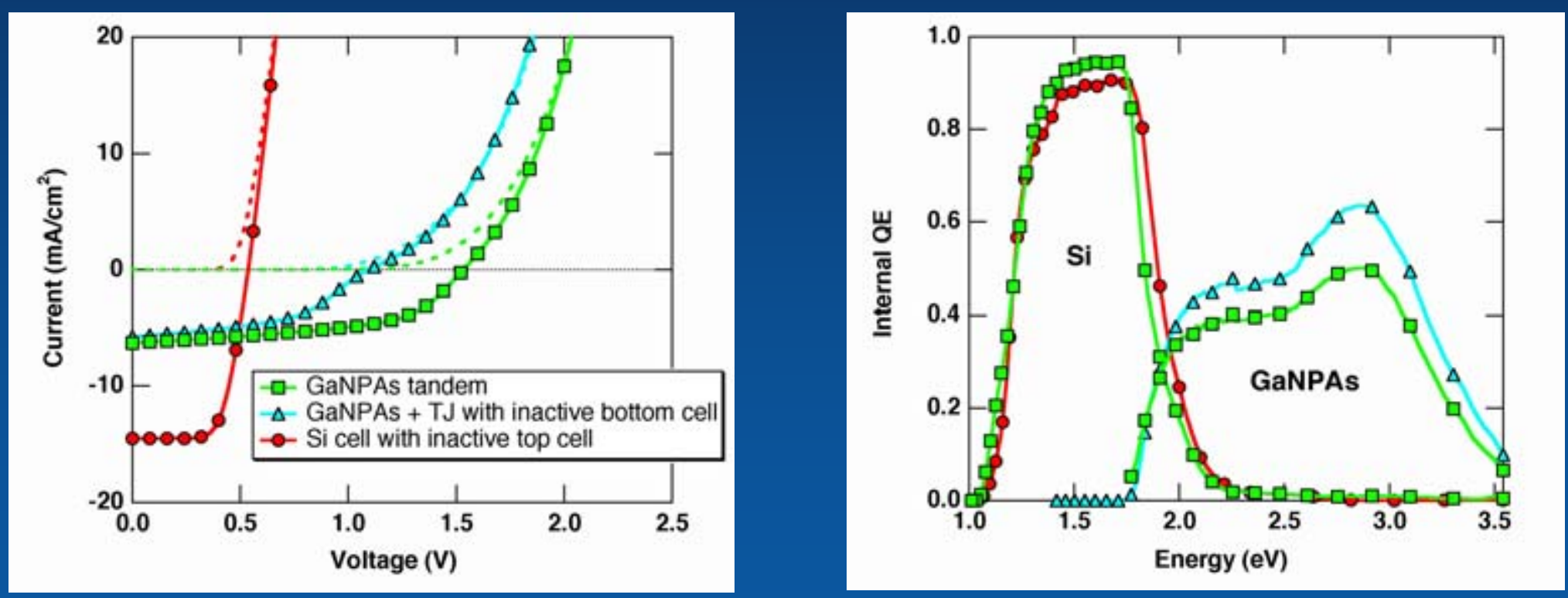

Geisz et al., PVSC 31 (2005) 695 


\section{Step Grading for Mismatch on GaP}

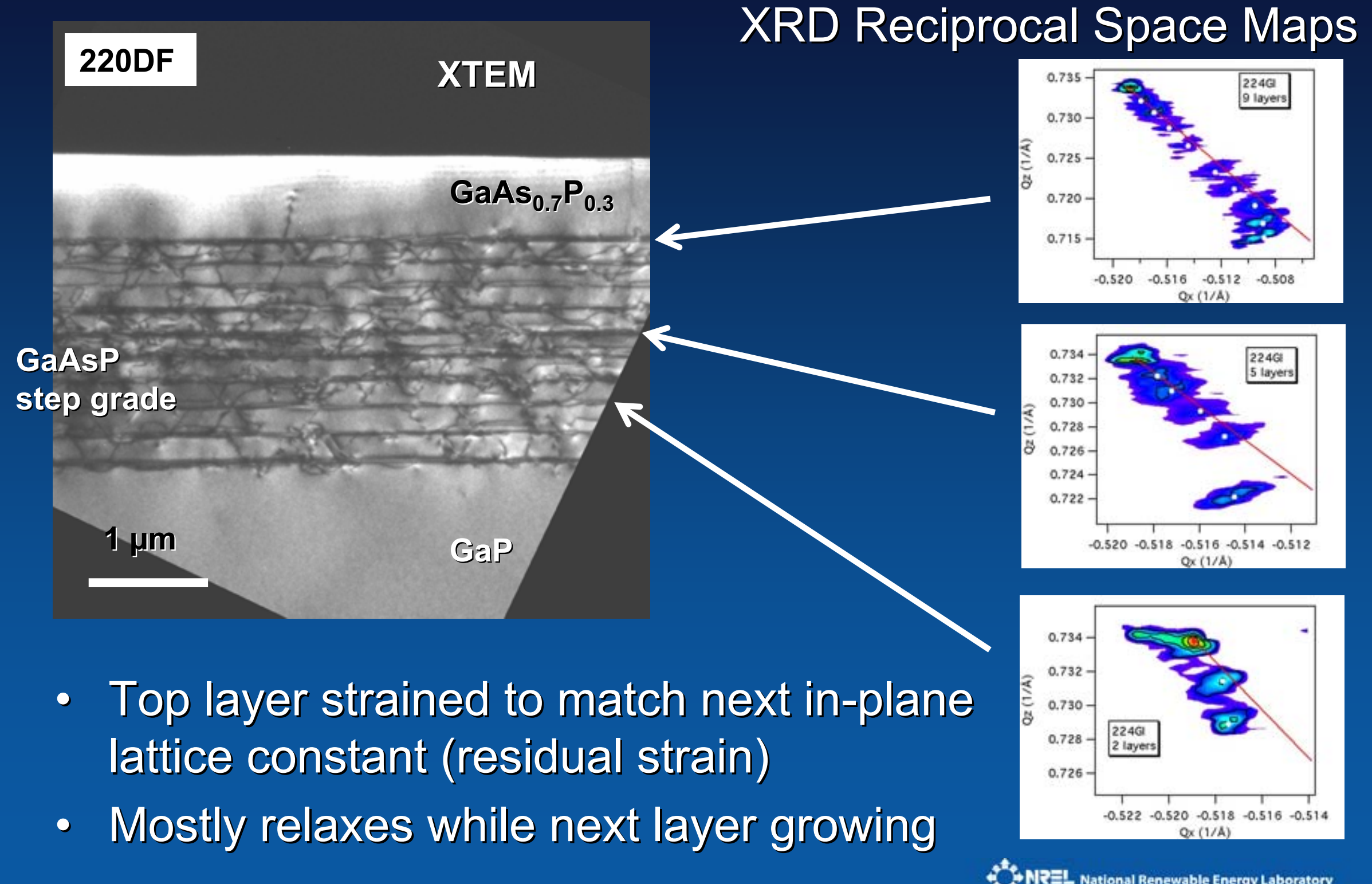




\section{Lattice-matched GaNP}

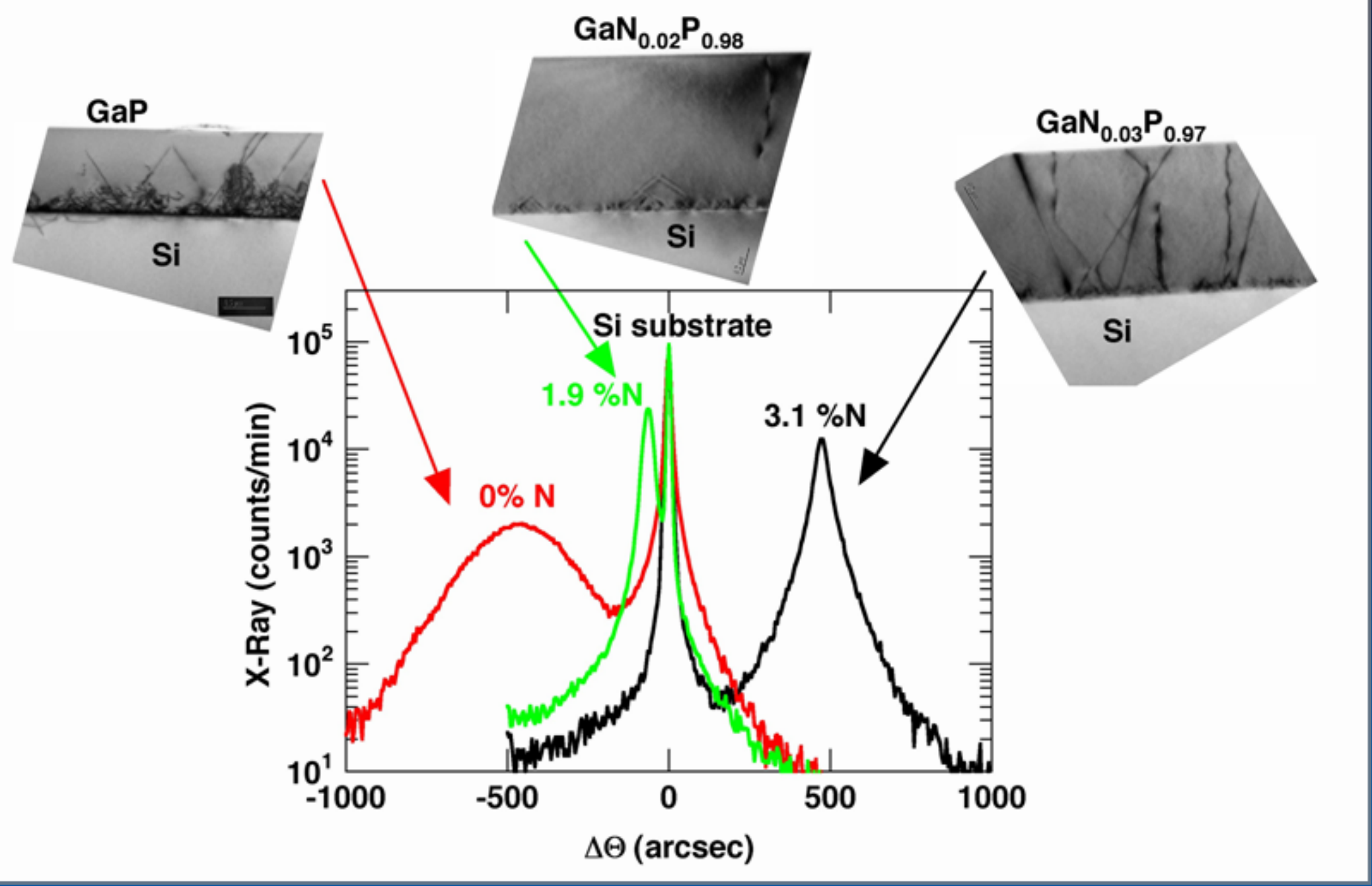




\section{GaNPAs/Si Tandem Solar Cell Structure}
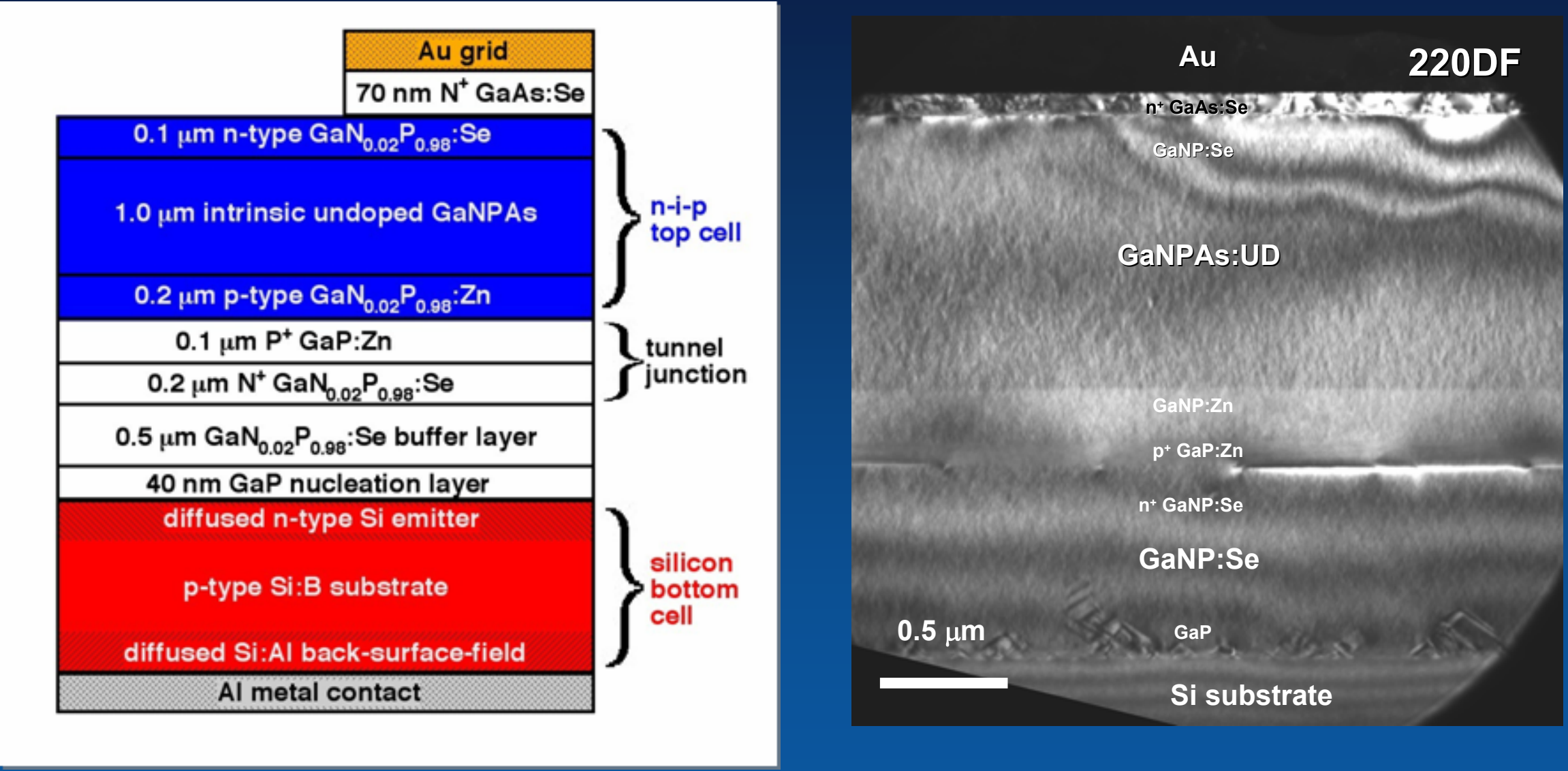


\section{GaAsP/Si Device Performance}
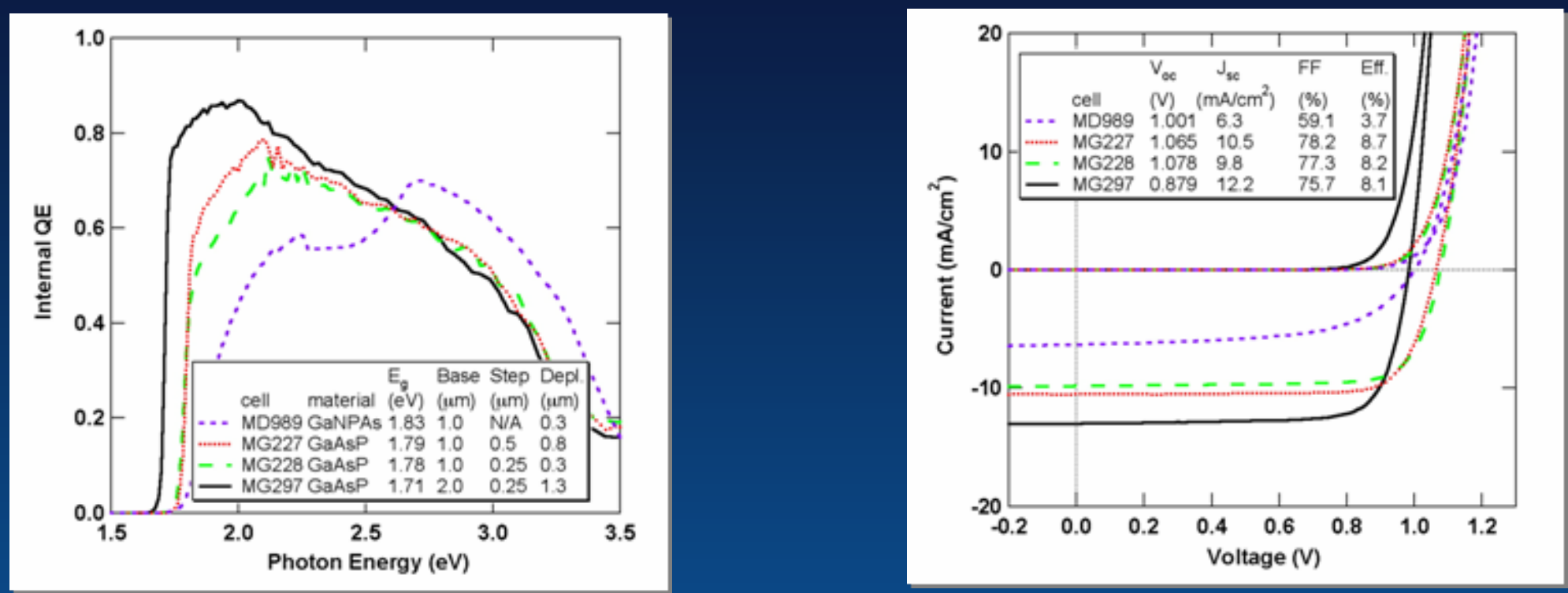

- Single-junction $8.7 \%$ efficiency AM1.5G w/o AR coat

- Increase $J_{s c}$ by lower $E_{g}$ (need about $20 \mathrm{~mA} / \mathrm{cm}^{2}$ for 2-junction)

- Improved QE with wide depletion region, but decent with thinner depletion region

- GaAsP/Si better than GaNPAs/GaP

- Longer diffusion length even with $10^{8} \mathrm{~cm}^{-2} \mathrm{TD}$ 